\title{
Metallicity Gradients in Globular Cluster Systems : the Trace of a Self-Enrichment Process?
}

\author{
G. Parmentier, P. Magain, A. Noels, A. Thoul \\ Institute of Astrophysics and Geophysics, University of Liège, avenue de \\ Cointe 5, B-4000 Liège, Belgium \\ E. Jehin
}

European Southern Observatory, Casilla 19001, Santiago 19, Chile

\begin{abstract}
We have developed a model of globular cluster self-enrichment, based on the ability of the globular cluster gaseous progenitors to retain the ejecta of a first generation of Type II Supernovae. The key point is that this ability depends on the pressure exerted on the progenitor cloud by the surrounding protogalactic medium and therefore on the location of the cloud in the protogalaxy. The model is able to explain the galactic halo metallicities and the metallicity gradient of the Old Halo which is thought to be the genuine galactic globular cluster system. The possibility that metallicity gradients are a common property of extragalactic globular cluster systems is also presented.
\end{abstract}

\section{The Galactic Halo Globular Cluster System}

From a model of galactic halo globular cluster (hereafter GC) self-enrichment, we reach the conclusion (Parmentier et al., 1999) that higher metallicities may be achieved in the inner galactic regions where the pressure of the protogalactic background, in which the GC primordial gaseous progenitors are embedded, is larger. A metallicity gradient throughout the galactic halo globular cluster system (hereafter GCS) should therefore be one of the traces of any early selfenrichment process.

The galactic halo does not exhibit a significant metallicity gradient. However, there have been growing evidences that the halo was partly built via accretions and mergers of satellite systems, each of them bearing their own GCs. As a result, the current halo GCS is partly composed of GCs born well apart from the original protoGalaxy, i.e. not indicative of the early formation of the galactic halo and of any self-enrichment process in GC primordial progenitor clouds.

If we consider the Old Halo subsystem, which may be the best image of the initial/genuine galactic halo GCS (Zinn 1993), then a significant metallicity gradient emerges from the observational data. Parmentier et al. (2000) show that a self-enrichment process can explain both the metallicity range exhibited by galactic halo GCs and the Old Halo metallicity gradient, especially when the model is combined with a hot protogalactic background pressure profile scaling as $P_{h} \propto D^{-2}$ (Murray \& Lin 1992). 


\section{Extragalactic Globular Cluster Systems}

One can now ask if metallicity gradients are a common property of GCSs. Most of the extragalactic GCSs exhibit colour gradients such that they are redder in the centre and bluer at larger radii. Since for old stellar systems $(\tau \geq 8 \mathrm{Gyr})$, broadband colours of GCs primarily reflect their metallicities (e.g. Worthey et al. 1994), these colour gradients are indicative of the presence of metallicity gradients. For instance, Geisler, Lee \& Kim (1996) found a radial metallicity gradient in the GCS of NGC4472 -the giant Virgo elliptical M49- of the order of $-0.4 \mathrm{dex} / \log (\mathrm{arcsec})$. Recent spectroscopic studies confirm this result (Beasley et al. 2000). These observations are therefore qualitatively in agreement with the self-enrichment model.

However, in most cases, these colour/metallicity gradients are an artefact coming from the bimodality of the metallicity distribution function (hereafter MDF) of the whole GCS. The gradient is driven by the smaller galactocentric distances of the GCs having redder colours/higher metallicities than the spatially more extended bluer/more metal-poor subgroup. Our Galaxy exhibits such a bimodality, with the MDF including two well-separated peaks, corresponding respectively to the disk/bulge population, metal-rich and centrally located, and to the halo population, metal-poor. Both groups present different kinematic properties as well (e.g. Zinn 1985). A similar situation is exhibited by the GCS of M31 (Barmby et al. 2000). Bimodal colour/metallicity distributions are also common for the GCSs of elliptical galaxies. These widespread bimodalities are the relic of two major phases of cluster formation in the early history of these galaxies. The clusters belonging to the most metal-rich peak of the MDF (inner population) most probably formed out of material chemically enriched by previous stellar generations (pre-enrichment). As such, to account for their formation is beyond the scope of our model since it describes the self-enrichment of initially primordial clouds. Any comparison between our model and observational data should be therefore limited to the blue/metal-poor population. That is the blue and red populations of clusters constitute two genuinely distinct subsystems is reinforced by some kinematic differences between both GC populations in some galaxies (see above for the Milky Way). For instance, in the case of NGC5128, Hui et al. (1995) present evidence that the blue (metal-poor) population does not rotate, while the red (metal-rich) population has significant rotation.

Beasley et al. (2000) try to isolate the two GC groups of NGC4472. They argue that both the red and blue populations show some evidence of a metallicity gradient, in agreement with the self-enrichment hypothesis. Some more studies devoted to the sole blue/metal-poor population of GCSs, determined both from photometric and kinematic data, in various types of galaxies and environments, are highly desirable in order to further test our self-enrichment model on an extragalactic scale.

Acknowledgments. This research was supported by contracts Pôle d'Attraction Interuniversitaire P4/05 (SSTC, Belgium) and FRFC F6/15-OLF63 (FNRS, Belgium) 


\section{References}

Barmby, P., Huchra, J.P., Brodie, J.P., Forbes, D.A., Schroder, L.L., \& Grillmair, C.J. 2000, AJ, 119, 727

Beasley, M.A., Sharples, R.M., Bridges, T.J., Hanes, D.A., Zepf, S.E., Ashman, K.M., \& Geisler, D. 2000, MNRAS, 318, 1249

Geisler, D., Lee, M.G., \& Kim, E. 1996, AJ, 111, 1529

Hui, X., Ford, H.C., Freeman, K.C., \& Dopita, M.A. 1995, ApJ, 449, 592

Murray S.D., Lin D.N.C. 1992, ApJ, 400, 265

Parmentier, G., Jehin, M., Magain, P., Neuforge, C., Noels, A., \& Thoul, A. 1999, A\&A, 352, 138

Parmentier, G., Jehin, M., Magain, P., Noels, A., \& Thoul, A. 2000, A\&A, 363, 526

Worthey G. 1994, ApJS, 95, 107

Zinn, R. 1985, ApJ, 293, 424

Zinn R., 1993 in ASP Conf. Ser. Vol. 48, The Globular Cluster - Galaxy Connection, ed. G.H. Smith \& J.P. Brodie, 38 